\title{
CVD Diamond Films - Synthesis, Microstructure, Applications
}

\author{
Wolfgang Jaeger
}

Institute for Materials Science, Christian Albrechts University Kiel, DE 24143 Kiel, Germany

Diamond is a material with extraordinary properties - electrically wide-band gap semiconducting and optical properties, high mechanical strength, high thermal conductivity, and biocompatibility. Single crystalline and nanocrystalline diamond thin films and diamond particle materials are utilized in a broad range of current applications and possess potential for novel future uses. Examples are high-power and high-temperature electronics, supercapacitors, single photon sources, microelectromechanical systems, sensor technology, diamond blades for medical surgery, functionalized nanoparticle materials for medical application, photocatalytic conversion of $\mathrm{CO}_{2}$ to chemicals and fuels, detector windows, electrodes for electrochemistry, surface coatings, tool coating, and abrasives. Diamond as 'supermaterial' is of high demand in industrial sectors, as diverse as oil and gas drilling, mining, road maintenance, stone and construction, wood-working, automotive, aerospace, and electronics.

Various techniques have been explored for the synthesis of diamond materials as particles, thin films and coatings. The synthesis of nanocrystalline and single crystalline diamond thin films by biasenhanced chemical vapor deposition (CVD) techniques has been investigated intensively in the past with the objective to understand nucleation and growth of textured and single-crystal diamond films on various types of substrates, such as silicon, silicon carbide, or cubic metal substrates. Despite the generally large lattice mismatch between diamond and the substrate materials, microwave-assisted CVD has been successful in the fabrication of thin diamond films of high structural quality and with outstanding properties. Applying transmission electron microscopy (TEM) and spectroscopy has proven to be successful in obtaining information on the microstructure of films, including interfaces, grain boundaries and crystal defects, and thus contributed to a solid understanding of the relationship between deposition parameters and structural and physical film properties. This brief review will describe early and more recent research on relevant aspects of the synthesis and microstructure of device-relevant CVD diamond films on various substrates. Some previous reviews of this subject may be found in $[1,2]$.

The advanced CVD process for diamond nucleation and growth on Si (001) substrates uses a narrow parameter window for the deposition of well-oriented diamond films, especially with respect to the choice of nucleation and kinetic conditions. A bias-enhanced nucleation step aims at forming a high density of diamond nuclei. Both the parameters chosen for ion bombardment and the kinetics play an important role, being determined by substrate temperature, gas (methane) concentration, process pressure, bias voltage, and nucleation time. For instance, the formation of misoriented crystals is influenced by ion bombardment induced defects and by re-nucleation of grains. Slightly different deposition conditions are utilized during the growth stage so that columnar growth of so called 'highlyoriented' grains with epitaxial orientation to the substrate is favored, and diamond grains with large misorientations become overgrown. During film deposition, the substrate temperatures are kept typically at about $800^{\circ} \mathrm{C}$ to $870^{\circ} \mathrm{C}$. The presence of boron in the gas phase was found to strongly enhance the stepflow lateral grain growth and to favour the lateral grain sizes [3]. Optimized diamond films are reproducibly grown nowadays by employing a bias-enhanced nucleation stage followed by a subsequent growth stage. 
The current knowledge about the microstructure of CVD diamond films results from extensive investigations for deposition on various crystalline substrates (e.g., $\mathrm{Si}, \mathrm{SiC}$, cubic $\mathrm{BN}, \mathrm{Cu}, \mathrm{Ni}$, and Ir). Deposition on $<001>$-oriented Si substrates, on $\beta$-SiC substrates, and on iridium appear to be most promising in forming thin films with a high degree of $<001>$ grain texture and orientation order, with lateral dimensions reaching several $10 \mu \mathrm{m}$, and with a high surface smoothness. Predominant remaining defects in the top layers of such films are small-angle grain boundaries [4]. Secondary carbon phases are largely absent for optimized conditions. Measured average polar and azimuthal spreads of grain orientations (as determined from X-ray rocking curves) of less than $1^{\circ}$ have been reported for diamond films on Ir [5]. TEM investigations of the interfaces show that misoriented diamond crystals are formed already during the nucleation stage of film formation, the major reason being the limited fraction of (001) facets on substrate surfaces roughened by hydrogen plasma etching [6, 7]. In direct epitaxy between diamond and $\mathrm{Si}$ (001), the interface is characterized by dense arrays of misfit dislocations [6]. For certain optimized deposition conditions, strain-relaxed (001) interfaces are achieved that are characterized by domains (several tens of nanometers in lateral dimension) with grooves along $<011>$ directions and with an interlayer of $\mathrm{SiC}$ (few nanometers in thickness) between diamond and $\mathrm{Si}$ [6]. The resulting improved physical film properties are exploited in technology for microsystems, microelectronics and medical applications. The synthesis of large-area (several inches) single-crystalline diamond films which would be of particular interest for applications in high-temperature microelectronics still remains a challenge.

The advanced characterization methods, made available through aberration-corrected (S)TEM at medium and low electron energies in the range between $300 \mathrm{keV}$ to $15 \mathrm{keV}$, are enabling studies of the microstructure on the atomic scale. Some examples are investigations of doping of diamond [8,9], and of the structure of diamond nanoparticles films and of their surfaces functionalized by molecules [10], to name but a few. A remaining challenge is the characterization of electron beam-sensitive materials, such as the characterization of diamond surface functionalization by molecules using imaging and spectroscopic investigations by (S)TEM methods under conditions of negligible electron beam damage. Another challenge is the imaging and spectroscopy of nitrogen-vacancy centers in diamond, being of interest for magnetometry and quantum science using electron spins, and the use of correlative TEMAPT characterization approaches [11].

\section{Selected References}

[1] C E Nebel et al (eds), Thin Film Diamond (Elsevier) Vol. 76 (2003)

[2] Srikanth, V., Jiang, X. (2011) Synthetic Diamond Films: Preparation, Electrochemistry, Characterization, and Applications (eds E. Brillas, C. A. Martínez-Huitle), John Wiley, Hoboken, USA

[3] X. Jiang, K. Schiffmann, C.-P.Klages, D. Wittorf, C. L. Jia, K. Urban, W. Jäger, J. Appl. Phys. 83 (1998) p 2511

[4] W. Jäger, D. Wittorf, C. L. Jia, K. Urban, Advances in Science and Technology 21 (1999) p 137

[5] T. Bauer et al., Diamond \& Related Materials 14 (2005) p 314

[6] D. Wittorf, W. Jäger, C. Dieker, A. Flöter, H. Güttler, Diamond and Related Mat. 9 (2000), 1696

[7] X. Jiang and C.L. Jia, Phys. Rev. Lett. 84 (2000) p 3658.

[8] J. Neethling et al., Diamond \& Related Materials 49 (2014) p 1

[9] S. Turner et al. Nanoscale 8 (2016) p 2212

[10] O. A. Williams, J. Hees, Ch. Dieker, W. Jäger, L. Kirste, C.E. Nebel, ACS NANO 4 (2010), 4824

[11] C Barroo, A Magyar, A Akey, D C Bell, Microscopy \& Microanalysis 22 S3 (2016), p. 694 